\title{
A massagem infantil como recurso para modulação do estresse em bebês em ambiente de cuidados coletivos
}

\author{
The infant massage as a resourse for modulating stress in infants in collective care environment \\ El masaje infatil como recurso para modulación del estrés en bebés en ambiente de cuidados colectivos
}

Telma Vitorina Ribeiro-Lima ${ }^{1}$, Lília lêda Chaves Cavalcante ${ }^{1}$.

\begin{abstract}
RESUMO
Objetivo: A presente revisão integrativa traz uma reflexão, fundamentada em evidências científicas, acerca dos potenciais benefícios das massagens infantis sobre a modulação do estresse em bebês de 0 a 6 meses de idade em ambientes de cuidados coletivos. Métodos: entre janeiro de 2007 e maio de 2018, a partir do portal dos Periódicos CAPES, foram levantados 64 artigos que contemplassem os objetivos deste estudo. Partindo-se destes, selecionou-se aqueles que avaliaram o estresse dos bebês com base em escalas de dor, marcadores bioquímicos e sinais vitais, e cujas descrições metodológicas descrevessem experimentos realizados especificamente em ambiente de cuidados coletivos, o que culminou em 18 artigos. Resultados: 0 único estudo ocorrido em creche era brasileiro e todos os demais, em hospitais, destacando-se as Unidades de Terapia Intensiva Neonatal (UTIN). Independentemente do tempo de duração das sessões e do período de aplicação das massagens, a maioria dos estudos indicaram mudanças nos indicadores de estresse nos bebês estudados. Os EUA foram o país com maior produtividade. A massagem de Tiffany Field se destacou dentre as técnicas ocidentais de massagem. Apenas dois estudos descreveram intervenções com massagens tradicionais: a Shantala (indiana) e a Yakson (coreana). Considerações Finais: os resultados dos estudos indicam que diversas técnicas de massagem infantil podem promover mudanças nos indicadores de estresse entre bebês em ambiente de cuidados coletivos. Novos estudos são necessários para explorar mais profundamente as massagens infantis originadas dos conhecimentos tradicionais dos povos. As lacunas observadas incentivam futuros estudos da mesma natureza em ambientes de creche e de acolhimento institucional.
\end{abstract}

Palavras-chave: Bebê, Recém-Nascido, Massagem, Creches, Hospitais Pediátricos.

\begin{abstract}
Objective: the present integrative review brings a scientifically based reflection on the potential benefits of infant massages on the modulation of stress in infants from 0 to 6 months of age in collective care settings. Methods: Between January 2007 and May 2018, from the CAPES Periodicals portal, 64 articles were included that contemplated the objectives of this study. From these, we selected those who assessed the stress of infants based on pain scales, biochemical markers and vital signs, and whose methodological descriptions described experiments performed specifically in a collective care environment, culminating in 18 articles. Results: the only study carried out in day-care centers was Brazilian and all the others, in hospitals, highlighting the Units of Neonatal Intensive Care (NICU). Regardless of the duration of the sessions and the period of application of the massages, most of the studies indicated changes in the stress indicators in the babies studied. The US was the country with the highest productivity. The Tiffany Field massage stood out among Western massage techniques. Only two studies described interventions with traditional massages: Shantala (Indian) and Yakson (Korean). Final considerations: the results of the studies indicate that several techniques of infant massage can promote changes in the indicators of stress among infants in a collective care setting. New studies are necessary to explore more deeply the infantile massages derived from the traditional knowledge of the people. The observed gaps encourage future studies of the same nature in nursery and institutional settings.
\end{abstract}

Key words: Baby, Infant, Massage, Foster Home Care, Pediatric Hospitals.

${ }^{1}$ Universidade Federal do Pará. Belém, Pará * E-mail: telmavitorina@gmail.com

Este estudo foi financiado pela Coordenação de Aperfeiçoamento de Pessoal de Nível Superior.

SUBMETIDO EM: 10/2018 


\section{RESUMEN}

Objetivo:La presente revisión integrativa trae una reflexión, fundamentada en evidencias científicas, acerca de potenciales beneficios de masajes infantiles sobre la modulación del estrés en bebés de 0 a 6 meses de edad en ambientes de cuidados colectivos. Métodos: Entre enero de 2007 y mayo de 2018, a partir del portal de Periódicos CAPES, se levantaron 64 artículos que contemplaran los objetivos de este estudio. A partir de estos, se seleccionaron aquellos que evaluaron el estrés de bebés con base en escalas de dolor, marcadores bioquímicos y signos vitales, y cuyas metodolgias describen experimentos realizados específicamente en ambiente de cuidados colectivos, lo que culminó en 18 artículos. Resultados: El único estudio ocurrido en guardería era brasileño y todos los demás, en hospitales, destacándose las Unidades de Terapia Intensiva Neonatal (UTIN). Independientemente del tiempo de duración de las sesiones y del período de aplicación de masajes, la mayoría de los estudios indicaron cambios en los indicadores de estrés en los bebés estudiados. Estados Unidos presentó la mayor productividad. El masaje de Tiffany Field se destacó entre las técnicas occidentales de masaje. Sólo dos estudios describieron intervenciones con masajes tradicionales: la Shantala (india) y la Yakson (coreana). Consideraciones finales: Los resultados de los estudios indican que diversas técnicas de masaje infantil pueden promover cambios en los indicadores de estrés entre bebés en ambiente de cuidados colectivos. Nuevos estudios son necesarios para explorar más profundamente los masajes infantiles originados de los conocimientos tradicionales de los pueblos. Las lagunas observadas incentivan futuros estudios de la misma naturaleza en ambientes de guardería y de acogida institucional.

Palabras clave: Bebé, Recien Nacido, Massaje, Guarderías Infantiles, Hospitales Pediátricos.

\section{INTRODUÇÃO}

Os mais diversos motivos podem afastar crianças do convívio parental e familiar, temporária ou definitivamente, seja pela necessidade dos pais deixarem os filhos em creches durante as jornadas de trabalho, ou por períodos de internação hospitalar das crianças e outras situações de vulnerabilidade da família (RAD et al., 2016; RANGEY \& SHETH, 2014), além dos motivos decorrentes de guerras, catástrofes naturais e orfandade. Todas essas situações têm em comum o afastamento das crianças dos cuidados e do afeto parental (AGER et al., 2011; IPEA, 2004; OLIVEIRA et al., 2012; OLIVEIRA et al., 2010; PICCININI et al., 2016), sendo a separação da família motivado por razões que combinam violência, negligência e pobreza, levando os pais a perderem, temporária ou definitivamente, a tutela de seus filhos para o Estado.

O tempo de duração e a qualidade do cuidado oferecido, em conjunto com os fatores inerentes a cada situação para o afastamento familiar, podem favorecer o estresse infantil, assim como repercutir de maneira importante no desenvolvimento global e na formação da personalidade dessas crianças (BOWLBY, 2004; MOURA e AMORIM, 2013; SPITZ, 2004; ZEANAH et al., 2003). Partindo deste pressuposto, a literatura científica tem apresentado estudos dedicados a compreender o desenvolvimento de bebês que se encontram sob cuidados coletivos, e que trazem propostas de intervenção que buscam proporcionar qualidade de vida e favorecer o desenvolvimento saudável dos mesmos.

Dentre essas propostas de intervenção, as várias técnicas de massagem infantil vêm ganhando destaque, uma vez que são evidentes os benefícios da sua aplicação para os bebês, como por exemplo, a promoção de seu crescimento biológico, tais como ganho de altura e de peso (RAD et al., 2016), melhor interação diádica entre mãe e bebê (FERBER et al., 2005), redução no comportamento de estresse, de níveis de cortisol (FOGAÇA et al., 2005; NEU et al., 2014), de agravos em saúde, ganho de peso e redução do tempo de permanência hospitalar (RAD et al., 2016; RANGEY \& SHETH, 2014).

O presente ensaio propõe uma reflexão, devidamente fundamentada por evidências científicas relatadas na literatura, sobre os potenciais benefícios que intervenções com massagens infantis podem proporcionar a bebês que se encontrem em ambiente de cuidados coletivos. $O$ enfoque da abordagem inclui medidas indicativas de estresse nesses bebês, sendo, basicamente: escalas de dor, marcadores bioquímicos de estresse e sinais vitais. Espera-se que os dados encontrados e aqui compartilhados possam contribuir para o desenho de novas estratégias de intervenção dentro dessa natureza, visando o benefício dessa população. 


\section{METODOLOGIA}

No período de janeiro de 2007 a maio de 2018, um primeiro levantamento foi realizado a partir do portal dos Periódicos CAPES, tendo os seguintes critérios de inclusão: (1) artigo que tivesse sido revisado por pares; (2) que tivesse sido publicado em inglês ou em português; (3) que descrevesse estudos experimentais; (4) que incluísse bebês nos seus objetivos (5) com faixa etária entre 0 e 6 meses; (6) que descrevesse intervenções com pelo menos uma técnica de massagem e/ou estimulação manual em bebês, tendo sido estes avaliados em função de critérios de saúde, desenvolvimento e/ou comportamento; (7) uma vez envolvendo outros tipos de terapia propostas como intervenções além da massagem, que cada uma delas tenha sido avaliada individualmente nos diferentes grupos de bebês participantes da pesquisa; (8) que englobasse dados não apenas dos adultos que aplicaram a massagem, mas sobretudo dos bebês.

Assim, neste levantamento inicial foram obtidos 64 artigos. Nele, um novo filtro foi aplicado em busca de estudos mais adequados ao objetivo da revisão integrativa proposta. Aos critérios acima descritos foram acrescentados os outros a seguir: (9) que o objetivo do estudo incluísse a avalição dos efeitos ou da eficácia da massagem sobre os bebês estudados; (10) que, de acordo com a metodologia, os bebês tivessem sido avaliados por escalas de dor, estresse bioquímico e sinais vitais; (11) que a metodologia tivesse descrito de forma clara que o experimento havia sido realizado em ambiente de cuidados coletivos, como creches, hospitais ou serviços de acolhimento. Quaisquer artigos que não contemplassem todos esses itens combinados foram excluídos da planilha de análise. A segunda seleção resultou em 18 artigos que serão apresentados a seguir, tendo sido um único em contexto de creche realizado no Brasil e todos os demais em contexto hospitalar, com destaque para as Unidades de Terapia Intensiva Neonatal (UTIN).

\section{RESULTADOS E DISCUSSÃO}

A Tabela 1 descreve os 18 artigos selecionados para o presente estudo, com seus autores e anos de publicação, locais onde foram realizados e suas distribuições de acordo com o uso de escalas de dor, marcadores bioquímicos do estresse e sinais vitais. Como se pode ver, 14 deles $(73,68 \%)$ avaliaram pelo menos um sinal vital, dentre os quais foram encontrados: frequência cardíaca ( $F C)$, frequência respiratória $(F R)$, pressão arterial (PA) e temperatura (T). O grupo de escalas de dor e o de marcadores bioquímicos do estresse, por sua vez, incluíram 5 (26,31\%) artigos cada um. Além disso, apenas 1 (5,3\%) artigo avaliou os três aspectos em conjunto, $1(5,3 \%)$ utilizou apenas a escala de dor, $3(15,8 \%)$ avaliaram escalas de dor e sinais vitais, $4(21 \%)$ avaliaram marcadores bioquímicos do estresse, e $10(52,63 \%)$ analisaram unicamente sinais vitais (Tabela 1).

Relativamente aos locais de realização dos estudos, 12 (63,2\%) ocorreram nos Estados Unidos (EUA), dentre os quais $4(21 \%)$ são de um único grupo de pesquisa em Miami, Estado da Florida. Os demais se distribuíram em outras localidades na proporção de 1 (5,3\%) estudo por país, dos quais foram: Brasil, Canadá, China, Coreia do Sul, Líbano e um sem informação sobre a localização. Ao longo dos anos de publicação, 2009 e 2014 se destacaram com, respectivamente, 5 e 3 publicações; seguidos de 2005 e 2013, com duas publicações cada um; nos demais anos registrou-se apenas 1 publicação por ano, com exceção de $2011 \mathrm{e}$ 2012, quando não houve publicações desta natureza (Tabela 1).

\section{Escalas de Dor}

O primeiro tópico abordará a avaliação de escalas de dor em bebês que vivem em ambientes de cuidados coletivos e que receberam algum tipo de massagem terapêutica. As escalas de dor encontradas no levantamento foram: escala de dor infantil neonatal (NIPS) (HATHAWAY et al., 2015; JAIN et al., 2006), escala de dor, agitação e sedação neonatal (N-PASS) (Livingston et al., 2009) e escala do perfil de dor infantil do prematuro (PIPP) (ABDALLAH et al., 2013; CHIK et al., 2017). Dos cinco estudos encontrados, todos ocorreram em contexto hospitalar, em ambiente de UTIN. Três estudos descreveram sua população como bebês (CHIK 
et al., 2017; HATHAWAY et al., 2015; LIVINGSTON et al., 2009) e dois especificaram como prematuros (ABDALLAH et al., 2013; JAIN et al., 2006).

Conforme é mostrado nas tabelas 1 e 2, apesar de terem ocorrido em países diferentes, e com onze anos de diferença entre um e outro, os estudos conduzidos no Canadá (JAIN et al., 2006) e na China (CHIK et al., 2017) apresentaram semelhanças nos objetivos, na metodologia e nos resultados. Ambos os estudos não incluíram grupo controle, pois os bebês serviram como seus próprios controles. Os autores dos dois estudos relataram benefícios da aplicação de massagem em bebês em relação à percepção da dor, concluindo que a massagem suave da perna antes da punção do calcanhar é segura e diminui as respostas de dor em prematuros (JAIN et al., 2006) e que bebês submetidos à punção venosa podem ser beneficiados na redução da percepção de dor pela massagem nos seus membros superiores (CHIK et al., 2017).

Dois outros estudos utilizaram grupo controle com bebês que não receberam massagem e que foram comparados com os grupos experimentais. No entanto, ambos se utilizaram de metodologias diferentes, que diferiram nas massagens aplicadas, no tempo de intervenção e nas escalas de dor utilizadas. Os resultados indicaram que bebês prematuros que receberam massagem de suas mães foram beneficiados com menor pontuação na escala de dor (ABDALLAH et al., 2013), enquanto que bebês que receberam massagem das enfermeiras apresentaram estabilidade fisiológica e nenhuma mudança na pontuação de agitação e dor (LIVINGSTON et al., 2009). No único estudo que avaliou escalas de dor e que não mencionou qualquer tipo de controle, observou-se melhora em resultados relacionados a dor, sugerindo que a massagem e o toque curativo podem ser terapias integrativas úteis no gerenciamento da dor em Unidades de Terapia Intensiva Neonatais (HATHAWAY et al., 2015).

Os dados apresentados apontam que massagens terapêuticas podem reduzir o sofrimento por dor entre bebês em ambiente de cuidados coletivos, de modo especial em ambiente de UTIN. Há também que se destacar o papel da mãe nesse processo, uma vez que a familiaridade da voz, do odor e do toque, e a própria vinculação de parentalidade podem potencializar a sensação de segurança e conforto no indivíduo que recebe a massagem, permeados pela liberação da ocitocina, conhecida como o hormônio do vínculo (MORHENN et al., 2012). De modo complementar, no tópico seguinte serão apresentados dados acerca dos efeitos da massagem dos marcadores bioquímicos do estresse em bebês em ambiente de cuidados coletivos.

\section{Marcadores bioquímicos do estresse}

Do presente levantamento, um total de quatro estudos avaliaram marcadores bioquímicos do estresse. Dois desses artigos descreveram sua população como bebês prematuros (IM \& KIM, 2009; JAIN et al., 2006), e dois como com bebês/neonatos saudáveis (FOGAÇA et al., 2005; WHITE-TRAUT et al., 2009). Dois desses estudos ocorreram em contexto hospitalar, em ambiente de UTIN (IM \& KIM, 2009; JAIN et al., 2006), enquanto que os outros dois ocorreram em ambiente de berçário, sendo um hospitalar (WHITE-TRAUT et al., 2009) e o outro de creche pública (FOGAÇA et al., 2005). Tais artigos estão dispostos na Tabela 2.

No estudo de Jain et al., (2006), mencionado anteriormente, bebês que receberam massagem de 2 minutos na perna homolateral ao braço puncionado para coleta sanguínea, antes ou após o procedimento, não apresentaram diferença significativa no cortisol sérico. Este estudo trabalhou com um único grupo de bebês, os quais serviram como seus próprios controles. Os três demais estudos executaram intervenções de massagem com duração de 15 minutos cada uma, sendo que dois descreveram continuidade nas aplicações no decorrer da semana seguinte. Em todos esses três estudos, os grupos experimentais apresentaram diferença significativa, tanto em relação aos grupos controles (IM \& KIM, 2009; WHITE-TRAUT et al., 2009) quanto ao longo do tempo (FOGAÇA et al., 2005). Este achado pode ser um indicativo de que o tempo de duração da massagem e a continuidade das aplicações por pelo menos uma semana são fatores relevantes para que se obtenha resultados significativos na modulação do eixo hipotálamo-pituitária-adrenal (HPA). 
Tabela 1 - Distribuição dos artigos de acordo com as variáveis relacionadas aos estresse pesquisadas.

\begin{tabular}{|c|c|c|c|c|c|}
\hline Artigo & Autor(es) & Local & Escalas de Dor & $\begin{array}{l}\text { Marcadores } \\
\text { Bioquímicos } \\
\text { de Estresse }\end{array}$ & Sinais Vitais \\
\hline 01 & Jain et al., 2006 & Calgary, Alberta, Canadá & $x$ & $x$ & $x$ \\
\hline 02 & Livingston et al., 2009 & Los Angeles, Califórnia, EUA & $x$ & & $x$ \\
\hline 03 & Abdallah et al., 2013 & Beirute, Líbano & $x$ & & \\
\hline 04 & Smith, S. L. et al., 2013 & Salt Lake, Utah, EUA & & & $x$ \\
\hline 05 & White - Traut et al., 2009 & Chicago, Illinois, EUA & & $x$ & \\
\hline 06 & Im \& Kim et al., 2009 & Coreia do Sul & & $x$ & \\
\hline 07 & Diego et al., 2009 & Miami, Florida, EUA & & & $x$ \\
\hline 08 & Diego et al., 2008 & Miami, Florida, EUA & & & $x$ \\
\hline 09 & Diego et al., 2014 & Miami, Florida, EUA & & & $x$ \\
\hline 10 & Massaro et al., 2009 & Washington DC & & & $x$ \\
\hline 11 & Yates et al., 2014 & Arkansas, EUA & & & $x$ \\
\hline 12 & Diego et al., 2005 & Miami, Florida, EUA & & & $x$ \\
\hline 13 & Smith, Jr et al., 2014 & EUA & & & $x$ \\
\hline 14 & Freitas et al., 2010 & Lisboa, Portugal & & & $x$ \\
\hline 15 & Fogaça (2005) & São Paulo, Brasil & & $x$ & \\
\hline 16 & Hathaway et al., 2015 & EUA & $x$ & & $x$ \\
\hline 17 & Rad et al., 2016 & Sem informação & & & $x$ \\
\hline 18 & Chik et al., 2017 & Hong Kong, China & $X$ & & $X$ \\
\hline
\end{tabular}

Fonte: Dados da pesquisa. 
Tabela 2 - Estudos com enfoque nos efeitos da massagem infantil sobre escalas de dor em bebês sob cuidados coletivos.

\begin{tabular}{|c|c|c|c|c|c|c|c|}
\hline $\begin{array}{l}\text { Autor(es) } \\
\text { /ano }\end{array}$ & $\begin{array}{l}\text { Intervenções aplica- } \\
\text { das nos grupos expe- } \\
\text { rimentais }\end{array}$ & Quem aplicou & $\begin{array}{c}\text { Tempo de } \\
\text { intervenção }\end{array}$ & $\begin{array}{l}\text { Aplica- } \\
\text { ções } \\
\text { diárias }\end{array}$ & $\begin{array}{l}\text { Período de } \\
\text { aplicação }\end{array}$ & Escalas de dor & Resultados \\
\hline $\begin{array}{l}\text { Jain et } \\
\text { al., } 2006\end{array}$ & $\begin{array}{l}\text { Massagem na perna } \\
\text { homolateral ao braço } \\
\text { puncionado; GE1: an- } \\
\text { tes da punção; GE2: } \\
\text { depois da punção }\end{array}$ & Pesquisador & 2 minutos & 1 única & 1 dia & $\begin{array}{l}\text { Escala de dor in- } \\
\text { fantil neonatal } \\
\text { (NIPS) }\end{array}$ & $\begin{array}{l}\text { Os escores da NIPS antes da punção do calcanhar eram } \\
\text { baixos, indicando ausência de dor nos bebês. A massa- } \\
\text { gem não foi associada a mudança significativa no NIPS. } \\
\text { Após punção de calcanhar, o NIPS aumentou em ambos } \\
\text { os grupos, mas a massagem não foi associada a um au- } \\
\text { mento significativamente maior na pontuação. }\end{array}$ \\
\hline $\begin{array}{l}\text { Livings- } \\
\text { ton et al., } \\
2009\end{array}$ & $\begin{array}{l}\text { GE: Massagem Infantil } \\
\text { (McClure, 2000) }\end{array}$ & $\begin{array}{l}\text { Mães; pais; } \\
\text { instrutor certifi- } \\
\text { cado de mas- } \\
\text { sagem infantil }\end{array}$ & 20 minutos & 1 diária & 7 dias & $\begin{array}{l}\text { Escala de dor, } \\
\text { agitação e seda- } \\
\text { ção neonatal (N- } \\
\text { PASS). }\end{array}$ & $\begin{array}{l}\text { Medidas comportamentais e fisiológicas infantis estavam } \\
\text { dentro dos limites de segurança durante as sessões de } \\
\text { massagem. }\end{array}$ \\
\hline $\begin{array}{l}\text { Abdallah } \\
\text { et al., } \\
2013\end{array}$ & $\begin{array}{l}\text { GE: Massagem tera- } \\
\text { pêutica infantil (Field et } \\
\text { al., 1987) }\end{array}$ & Mãe & 10 minutos & 1 & $\begin{array}{l}\text { Até alta hos- } \\
\text { pitalar }\end{array}$ & $\begin{array}{l}\text { Escala do perfil } \\
\text { de dor infantil do } \\
\text { prematuro } \\
\text { (PIPP) }\end{array}$ & $\begin{array}{l}\text { Bebês massageados apresentaram significativamente } \\
\text { menor pontuação no teste PIPP após teste do pezinho } \\
\text { quando comparado com antes da massagem, e apresen- } \\
\text { taram menor pontuação de PIPP no momento da alta } \\
\text { quando comparados ao grupo controle. }\end{array}$ \\
\hline $\begin{array}{l}\text { Hatha- } \\
\text { way et } \\
\text { al., } 2015\end{array}$ & $\begin{array}{l}\text { GE1: MI+TC ou outro } \\
\text { TE; GE2: apenas MI } \\
\text { GE3: apenas TC } \\
\text { GE4: outra terapia }\end{array}$ & $\begin{array}{l}\text { Enfermeira es- } \\
\text { pecialista em } \\
\text { saúde holística }\end{array}$ & 35 minutos & -- & -- & $\begin{array}{l}\text { Escala de dor in- } \\
\text { fantil neonatal } \\
\text { (NIPS) }\end{array}$ & $\begin{array}{l}\text { Os escores de dor foram significativamente menores nos } \\
\text { bebês que receberam massagem, inclusive no momento } \\
\text { da alta hospitalar }\end{array}$ \\
\hline $\begin{array}{l}\text { Chik et } \\
\text { al., } 2017\end{array}$ & $\begin{array}{l}\text { Massagem no braço } \\
\text { GE1: } 1^{\circ} \text { massagem, } 2^{\circ} \\
\text { protocolo de controle } \\
\text { GE2: vice-versa }\end{array}$ & $\begin{array}{l}\text { Enfermeira ex- } \\
\text { periente de } \\
\text { UTIN treinada }\end{array}$ & 2 minutos & 1 & 1 dia & $\begin{array}{l}\text { Escala de Perfil } \\
\text { de dor infantil do } \\
\text { prematuro } \\
\text { (PIPP) }\end{array}$ & $\begin{array}{l}\text { Valores médios de dor foram significativamente menores } \\
\text { nos bebês que receberam massagem, quando compara- } \\
\text { dos com aqueles que não receberam massagem }\end{array}$ \\
\hline $\begin{array}{l}\text { White - } \\
\text { Traut et } \\
\text { al., } 2009\end{array}$ & $\begin{array}{l}\text { GE1: estimulação mul- } \\
\text { tissensorial } \\
\text { GE2: estimulação ape- } \\
\text { nas tátil }\end{array}$ & Pesquisador & 15 minutos & -- & -- & Cortisol salivar & $\begin{array}{l}\text { Declínio constante significativo nos níveis de cortisol, ao } \\
\text { contrário dos bebês que receberam apenas estimulação } \\
\text { tátil ou nenhuma estimulação e tiveram seus níveis de } \\
\text { cortisol elevados }\end{array}$ \\
\hline $\begin{array}{l}\operatorname{Im} \& \\
\text { Kim, } \\
2009\end{array}$ & $\begin{array}{l}\text { GE1: massagem Yak- } \\
\text { son; GE2: toque gentil }\end{array}$ & $\begin{array}{l}\text { Enfermeiras } \\
\text { pesquisadoras } \\
\text { treinadas } \\
\text { (EPT) }\end{array}$ & 15 minutos & 2 & $\begin{array}{l}7 \text { dias con- } \\
\text { secutivos } \\
\text { por } 15 \text { dias }\end{array}$ & $\begin{array}{l}\text { Cortisol urinário } \\
\text { e norepinefrina } \\
\text { urinária }\end{array}$ & $\begin{array}{l}\text { Após o período de intervenção, bebês nos grupos Yakson } \\
\text { e THG tiveram níveis significativamente mais baixos de } \\
\text { hormônio do estresse (cortisol urinário e norepinefrina uri- } \\
\text { nária) em comparação com os do grupo controle. Ne- } \\
\text { nhuma diferença significativa foi encontrada em níveis de } \\
\text { hormônio do estresse entre os bebês dos grupos Yakson } \\
\text { e THG. }\end{array}$ \\
\hline $\begin{array}{l}\text { Fogaça, } \\
2005\end{array}$ & GE: Shantala & Autora & 15 minutos & 2 & $\begin{array}{l}2 \text { dias con- } \\
\text { secutivos e } \\
1 \text { semana } \\
\text { depois (ma- } \\
\text { nhã e tarde) }\end{array}$ & Cortisol salivar & $\begin{array}{l}\text { Após a aplicação da massagem houveram alterações sig- } \\
\text { nificativas nos níveis de cortisol, principalmente nos perí- } \\
\text { odos da manhã e à tarde, sugerindo possível adaptação } \\
\text { do eixo adrenal-ptuitário-hipotalâmico }\end{array}$ \\
\hline
\end{tabular}

Fonte: Dados da pesquisa. MI: massagem infantil; TC: toque curativo; TE: trabalho de energia; GE: grupo experimental.

REAS/EJCH | Vol. 11 (1) | e438 | DOI: https://doi.org/10.25248/reas.e438.2019 Página 6 de 12 
É interessante destacar, ainda, o estudo que demonstrou que a estimulação multissensorial obteve bons resultados na redução do estresse de recém nascidos saudáveis, ao passo que a estimulação apenas tátil, pelo contrário, foi associada ao aumento dos níveis de cortisol nesta última (WHITE-TRAUT et al., 2009). A estimulação multissensorial não envolve apenas a tátil, mas também a visual, a auditiva e a vestibular, solicitando que o a pessoa que a aplica esteja mais envolvida na interação com o bebê. Esse envolvimento é o mesmo que se espera de alguém que aplica a massagem Shantala em um bebê, em que se ultrapassa a simples estimulação tátil com óleo, e encoraja o estreitamento do vínculo da pessoa que a aplica com a criança que a recebe, esperando-se que tal postura pode promover um melhor desenvolvimento psíquico emocional dessas crianças (LEBOYER, 1995). O estudo de Fogaça (2005) reforça essa proposição, uma vez que demonstrou que a Shantala foi capaz de proporcionar uma adaptação no eixo HPA de bebês em uma creche pública ao longo do período de estudo. Na sequência, o próximo tópico abordará a avaliação dos sinais vitais em bebês em ambiente de cuidados coletivos que recebem algum tipo de massagem terapêutica.

\section{Sinais vitais}

Um total de dez estudos do presente levantamento avaliaram os efeitos de massagens terapêuticas sobre os sinais vitais de bebês em ambiente de cuidados coletivos. Destes, cinco trabalharam com dois grupos, sendo um grupo experimental que recebeu algum tipo de massagem e um grupo controle que não recebeu (DIEGO et al., 2008; FREITAS et al., 2010; LIVINGSTON et al., 2009; J. R. SMITH et al., 2014; S. L. SMITH et al., 2013). Dois estudos apresentaram dois grupos experimentais com aplicações distintas de massagem e mais um grupo controle que não recebeu massagem (DIEGO et al., 2005, 2009). Por fim, três desses estudos trabalharam apenas com grupos experimentais, sem grupos controles para comparar (DIEGO et al., 2014; HATHAWAY et al., 2015; YATES et al., 2014). A Tabela 03 apresenta estes estudos comparados de acordo com os autores e ano de publicação, as técnicas de massagem aplicadas, as pessoas escolhidas para aplicar a massagem, o tempo de duração da intervenção, o número de aplicações diárias, o período de aplicação, sinais vitais pesquisados e resultados obtidos.

Todos os 10 artigos que avaliaram sinais vitais foram realizados em contexto hospitalar, em ambiente de Unidade de Terapia Intensiva Neonatal (UTIN). Os estudos de Livingston et al., (2009) e de Hathaway et al., (2015) descreveram suas populações apenas como bebês, enquanto todos os demais definiram suas populações como bebês ou neonatos prematuros. Os sinais vitais descritos nesses estudos foram: frequência cardíaca $(F C)$, frequência respiratória $(F R)$, pressão arterial $(P A)$, temperatura $(T)$ e saturação de oxigênio (Sat. O2). A distribuição desses estudos conforme avaliação dos sinais vitais de bebês em ambiente de cuidados coletivos que receberam massagem terapêutica está apresentada no gráfico 01 . Dos dez estudos, sete apresentaram algum tipo de mudança nos padrões de sinais vitais, dois referiram não ter havido mudanças estatisticamente significativas e um referiu estabilidade das medidas fisiológicas no decorrer das sessões. A massagem terapêutica infantil de Tiffany Field ganhou destaque entre esses estudos, estando presente em cinco deles. Tais resultados serão discutidos e comparados a seguir.

Dois estudos apresentaram metodologias semelhantes com resultados diferentes, de modo que aplicaram uma única sessão de massagem em um único dia, no entanto, enquanto um registrou diferenças significativas o outro registrou o contrário. Ambos os estudos utilizaram variações da mesma técnica, a massagem terapêutica infantil de Tiffany Field: Diego et al. (2009) utilizou a versão com pressão moderada (FIELD et al., 2007), ao passo que Yates et al. (2014) se embasou na versão original (FIELD et al., 1986) apenas retirando a estimulação cinestésica. Em busca de uma explicação para isso, verificou-se que os objetivos de cada um dos estudos foram essenciais no direcionamento dos resultados obtidos. Destaque-se, ainda, que o primeiro estudo incluiu um grupo controle em suas análises, recurso que não foi adotado pelo segundo. 
Tabela 3 - Estudos com enfoque nos efeitos da massagem infantil sobre sinais vitais de estresse em bebês sob cuidados coletivos.

\begin{tabular}{|c|c|c|c|c|c|c|c|}
\hline $\begin{array}{l}\text { Autor(es) } \\
\text { /ano }\end{array}$ & $\begin{array}{c}\text { Massagem aplicada no grupo } \\
\text { experimental }\end{array}$ & Quem aplicou & $\begin{array}{l}\text { Tempo de } \\
\text { interven- } \\
\text { ção }\end{array}$ & $\begin{array}{l}\text { Aplica- } \\
\text { ções di- } \\
\text { árias }\end{array}$ & $\begin{array}{l}\text { Período de } \\
\text { aplicação }\end{array}$ & $\begin{array}{l}\text { Sinais } \\
\text { Vitais }\end{array}$ & Resultados \\
\hline $\begin{array}{l}\text { Livingston et } \\
\text { al., } 2009\end{array}$ & $\begin{array}{l}\text { Massagem infantil (McClure, } \\
\text { 2000) }\end{array}$ & $\begin{array}{l}\text { Mães; pais; instrutor } \\
\text { certificado de massa- } \\
\text { gem infantil. }\end{array}$ & 20 minutos & -- & $\begin{array}{l}7 \text { dias con- } \\
\text { secutivos }\end{array}$ & $\begin{array}{l}\mathrm{FC}, \mathrm{FR}, \mathrm{O} 2 \\
\text { e PA }\end{array}$ & $\begin{array}{l}\text { Medidas comportamentais e fisiológicas infan- } \\
\text { tis foram descritas como estando dentro dos li- } \\
\text { mites de segurança durante as sessões de } \\
\text { massagem. }\end{array}$ \\
\hline $\begin{array}{l}\text { Smith, S. L. } \\
\text { et al., } 2013\end{array}$ & $\begin{array}{l}\text { Protocolo de massagem infantil } \\
\text { USA modificado }\end{array}$ & $\begin{array}{l}\text { Massoterapeutas licen- } \\
\text { ciados e certificados } \\
\text { em massagem infantil }\end{array}$ & 20 minutos & 2 & $\begin{array}{c}29 \text { dias } \\
\text { (4 semanas) }\end{array}$ & VFC & $\begin{array}{l}\text { GE: com aumento na VFC } \\
\text { GC: sem aumento na VFC } \\
\text { Melhora na VFC entre os meninos do GE } \\
\text { quando comparados às meninas. }\end{array}$ \\
\hline $\begin{array}{l}\text { Diego et al., } \\
2008\end{array}$ & $\begin{array}{l}\text { Massagem infantil (sem referên- } \\
\text { cia) }\end{array}$ & Terapeutas treinados & 15 minutos & 3 & -- & $\begin{array}{l}\text { Tempera- } \\
\text { tura }\end{array}$ & $\begin{array}{l}\text { Maior aumento na temperatura no GE quando } \\
\text { comparados ao GC }\end{array}$ \\
\hline $\begin{array}{l}\text { Smith, Jr et } \\
\text { al., } 2014\end{array}$ & Massagem Técnica M & $\begin{array}{l}\text { Investigador principal } \\
\text { ou um membro da } \\
\text { equipe de pesquisa }\end{array}$ & 7 minutos & 6 & 5 semanas & $\begin{array}{l}\text { FC, FRe } \\
\text { O2 }\end{array}$ & $\begin{array}{l}\text { Houveram diferenças significativas ao final do } \\
\text { estudo. }\end{array}$ \\
\hline $\begin{array}{l}\text { Freitas et al., } \\
2010\end{array}$ & Massagem de Tiffany Field & $\begin{array}{l}31 \text { enfermeiras treina- } \\
\text { das }\end{array}$ & 15 minutos & 3 & 5 dias & $\begin{array}{l}\text { FC, FR, O2, } \\
\text { PA e tem- } \\
\text { peratura }\end{array}$ & $\begin{array}{l}\text { Não houve diferença estatisticamente signifi- } \\
\text { cante entre os grupos. }\end{array}$ \\
\hline $\begin{array}{l}\text { Diego et al., } \\
2009\end{array}$ & $\begin{array}{l}\text { Massagem com pressão mode- } \\
\text { rada (Field, Diego, \& Hernan- } \\
\text { dez-Reif, 2007). }\end{array}$ & Terapeutas & 15 minutos & 1 & -- & $\mathrm{FC}$ & $\begin{array}{l}\text { Pressão moderada: - menor aumento da FC, } \\
\text { sugerindo atenuação da dor; - retorno mais rá- } \\
\text { pido aos valores da linha de base, sugerindo } \\
\text { melhor recuperação após o estresse. }\end{array}$ \\
\hline $\begin{array}{l}\text { Diego et al., } \\
2005\end{array}$ & $\begin{array}{l}\text { Massagem com pressão mode- } \\
\text { rada (Field et al., 1986) }\end{array}$ & Vários terapeutas & 15 minutos & 3 & 5 dias & $\mathrm{FC}$ & $\begin{array}{l}\text { Aumento significativo do tônus vagal, ambos } \\
\text { com pico durante a massagem, apenas no } \\
\text { grupo experimental }\end{array}$ \\
\hline $\begin{array}{l}\text { Diego et al., } \\
2014\end{array}$ & $\begin{array}{l}\text { GE1: estimulação tátil (ET) (Fi- } \\
\text { eld et al., 1986) } \\
\text { GE2: estimulação cinestésica } \\
\text { (EC) (Moyer-Mileur et al., 2000) }\end{array}$ & Pesquisador treinado & 10 minutos & 3 & 5 dias & FC & $\begin{array}{l}\text { Atividade vagal aumentou significativamente } \\
\text { durante ET e diminuiu significativamente du- } \\
\text { rante EC, associando a massagem ao au- } \\
\text { mento da atividade vagal. }\end{array}$ \\
\hline $\begin{array}{l}\text { Yates et al., } \\
2014\end{array}$ & $\begin{array}{l}\text { Massagem de Field et al., } 1986 \\
\text { GE1: primeiro dia; GE2: se- } \\
\text { gundo dia }\end{array}$ & 2 fisioterapeutas & 10 minutos & 1 única & 1 dia & $\mathrm{FC} \mathrm{e} \mathrm{O} 2$ & $\begin{array}{l}\text { Não houve mudanças significativas na fre- } \\
\text { quência cardíaca e na saturação de oxigênio } \\
\text { durante a massagem terapêutica. }\end{array}$ \\
\hline $\begin{array}{l}\text { Hathaway et } \\
\text { al., } 2015\end{array}$ & $\begin{array}{l}\text { GE1: massagem e toque cura- } \\
\text { tivo ou outro trabalho de ener- } \\
\text { gia; GE2: somente massagem; } \\
\text { GE3: somente toque curativo } \\
\text { GE4: outra terapia única }\end{array}$ & $\begin{array}{l}\text { Enfermeira especialista } \\
\text { em saúde holística }\end{array}$ & $\begin{array}{l}35 \text { minutos } \\
\text { (média) }\end{array}$ & -- & -- & $\mathrm{FC} \mathrm{e} \mathrm{O} 2$ & $\begin{array}{l}\text { FC e saturação de } \mathrm{O} 2 \text { mostraram diferenças } \\
\text { significativas entre as medidas feitas antes e } \\
\text { após a intervenção, com aumento da primeira } \\
\text { e redução da segunda }\end{array}$ \\
\hline
\end{tabular}

Fonte: Dados da pesquisa. GE (grupo experimental); GC (grupo controle); FC (frequência cardíaca); FR (frequência respiratória); VFC (variabilidade de frequência cardíaca); O2 (saturação de oxigênio); PA (pressão arterial).

REAS/EJCH | Vol. 11 (1) | e438 | DOI: https://doi.org/10.25248/reas.e438.2019 Página 8 de 12 
Gráfico 1 - Distribuição dos estudos conforme avaliação dos sinais vitais de bebês em ambiente de cuidados coletivos que receberam massagem terapêutica.

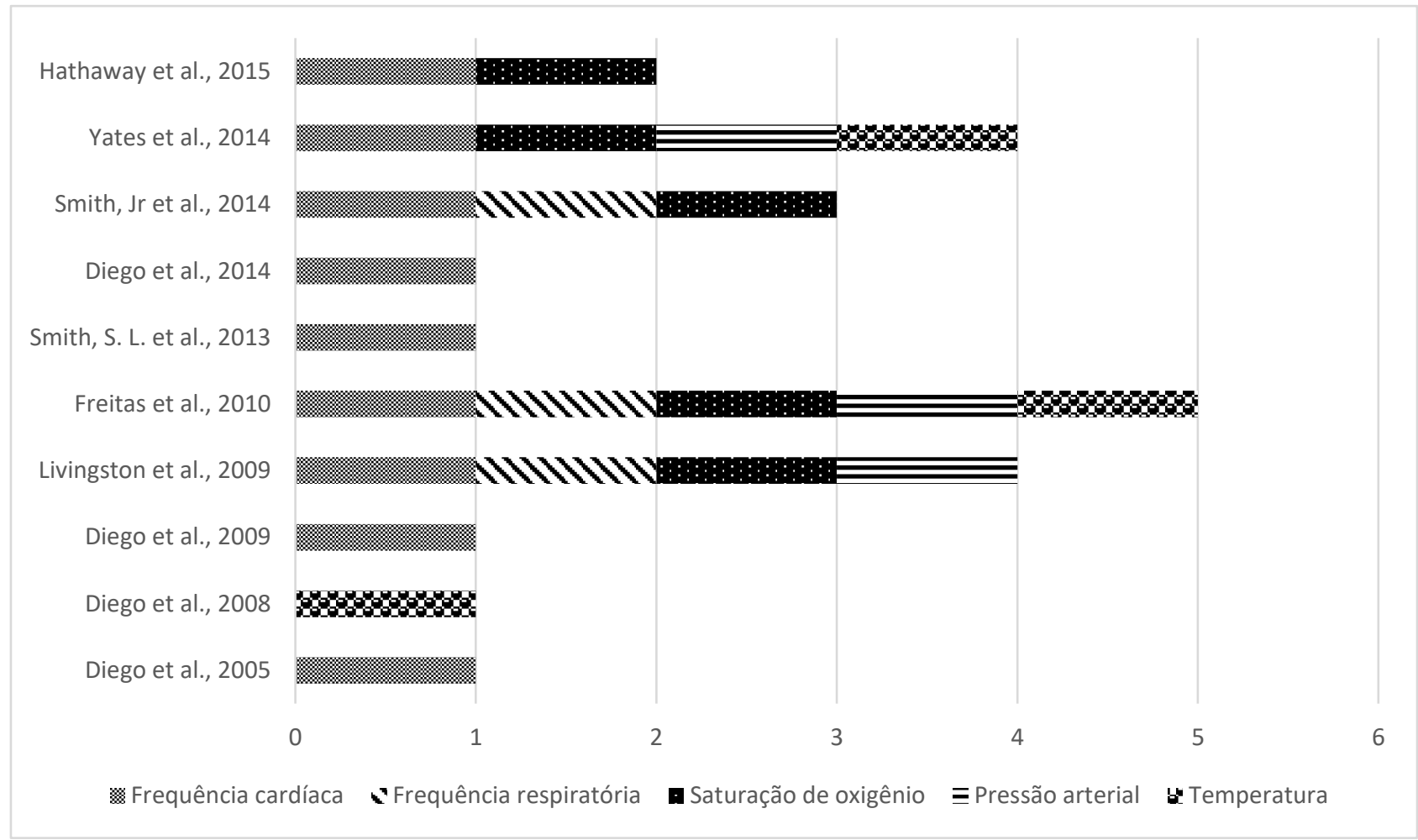

Fonte: Dados da pesquisa.

O estudo que apresentou diferenças significativas tinha como objetivo verificar se a massagem teria um efeito de atenuação da dor em bebês no momento da remoção de fita cirúrgica da pele dos bebês, de modo a envolver no contexto um agente estressor. Por outro lado, o estudo que registrou não haver mudanças significativas tinha como objetivo verificar a eficiência da massagem na indução do sono dos bebês. Deste modo, é possível considerar que uma única massagem pode promover mudanças na frequência cardíaca, podendo indicar atenuação da dor e redução do estresse; porém não sendo suficiente para modificar nem frequência cardíaca, nem saturação de oxigênio em situações que não envolvem agentes estressores agudos. Entretanto, para se verificar essa hipótese, novos estudos devem ser propostos e executados.

Os outros três estudos que realizaram a massagem de Tiffany Field utilizaram tempos iguais ou similares, tanto para duração das sessões como para período de aplicação. Destes, dois foram realizados em unidades de terapia intensivas neonatais (UTIN), em Miami, Florida; e, como resultados, apresentaram aumento significativo do tônus vagal. Ambos os estudos compararam dois grupos experimentais, porém apenas um dispôs de grupo controle (DIEGO et al., 2005, 2014).

O terceiro estudo foi realizado em unidades de cuidados intermediários (UCI) neonatal em Lisboa, Portugal, e comparou o grupo experimental ao controle. Não foram registradas diferenças estatisticamente significativas em relação aos sinais vitais mensurados. Esses dados podem indicar uma maior vulnerabilidade fisiológica dos bebês em UTI seja fator relevante para a maior variabilidade da frequência cardíaca, se comparados com bebês em estado menos grave em UCI. No entanto, novos estudos são necessários para que um maior aprofundamento traga luz sobre esse debate (FREITAS et al., 2010).Dos cinco estudos restantes, apenas um não apontou diferenças, seja entre o grupo experimental e o grupo controle, ou no decorrer do tempo. No entanto, este estudo descreveu estabilidade fisiológica dos bebês avaliados, estando os valores de sinais vitais dentro dos limites de segurança no decorrer das aplicações. Deste modo, os resultados descritos contemplaram o objetivo do estudo que era verificar a viabilidade e a segurança de um programa de massagem para bebês em condições médicas complexas (LIVINGSTON et al., 2009). 
Houve um único estudo que avaliou apenas temperatura e referiu aumento da mesma no grupo experimental, quando comparado ao grupo controle. Nele, o tempo de intervenção e o número de aplicações diárias apresentaram similaridade com outros estudos, ainda que o período de aplicações não tenha ficado claro no texto, deixando uma lacuna em um aspecto importante para comparações com outras pesquisas (DIEGO et al., 2008). Por outro lado, os dois estudos que referiram tempo de aplicação mais estendido, como 4 a 5 semanas, relataram, respectivamente, diferenças entre grupo experimental e grupo controle, e diferenças ao final do estudo (J. R. SMITH et al., 2014; S. L. SMITH et al., 2013). Por fim, um estudo que envolveu massagem infantil e toque curativo distribuídos em quatro grupos experimentais sem grupo controle registrou diferenças significativas antes e após as intervenções, ainda que não tenha esclarecido quantas aplicações diárias e qual o período de aplicação (HATHAWAY et al., 2015).

Com base nos dados apresentados, dentre os indicadores de estresse avaliados para o presente estudo, sinais vitais estiveram presentes em $14(73,68 \%)$ do total de publicações, sendo que $10(52,62 \%)$ do mesmo total não incluíram indicadores de outra natureza, tais como escalas de dor e marcadores fisiológicos do estresse. É possível que este perfil se deva à maior acessibilidade aos dispositivos de medição de sinais vitais no ambiente hospitalar, onde ocorreram 94,4\% (17/18) dos estudos. Por sua vez, os dois outros grupos de indicadores apresentaram menor frequência provavelmente por necessitarem de logística não diretamente associada à rotina hospitalar, mas que, ao contrário, dependiam da colaboração de outros setores. Se as escalas de dor deveriam ser aplicadas por profissionais capacitados, por sua vez, os marcadores bioquímicos do estresse deveriam ser determinados por exames laboratoriais.

Independentemente do tempo de duração de cada sessão e do período de aplicação das massagens, a maioria dos estudos avaliados indicaram mudanças nos indicadores de estresse em bebês vivendo em ambiente de cuidados coletivos. Aqueles que não apresentaram mudanças também podem ser úteis à comparação com os primeiros, além de possibilitar refletir sobre as diferenças nas metodologias aplicadas. A análise e a comparação cuidadosas dessas metodologias podem auxiliar no desenho de novos estudos que tenham como objetivo a avaliação dos efeitos de massagens terapêuticas em crianças vivendo em ambiente de cuidados coletivos.

Ainda que os trabalhos do presente levantamento tenham contemplado tantos países ocidentais como orientais, os EUA foi o país que mais se destacou em termos de produção. E dentre as publicações estadunidenses destacou-se o grupo de pesquisa de Miami, Flórida, mostrando que a técnica de Tiffany Field já se tornou uma referência ocidental enquanto massagem terapêutica voltada para o público infantil. As suas contrapartes orientais, por outro lado, como a indiana Shantala ou a coreana Yakson, que são tradicionalmente conhecidas e praticadas em seus países, ainda precisam ter seus benefícios devidamente explorados por novos estudos.

A maioria dos estudos foi realizada em âmbito hospitalar, sendo apenas um em ambiente de creche. Esta preferência provavelmente se deu pela maior facilidade de acesso aos instrumentos de medição dos indicadores, do treinamento das pessoas que aplicariam as técnicas de massagem (em sua maioria profissionais do quadro de funcionários) e de controle ambiental dos sujeitos de pesquisa (os bebês). Esse perfil demonstra a necessidade de se expandir estudos dessa natureza para outros ambientes de cuidados coletivos, pensando-se em explorar não apenas as creches, mas também espaços de acolhimento institucional de crianças em situação de vulnerabilidade, que tenham sido vítimas da pobreza, negligência e/ou violência.

\section{CONSIDERAÇÕES FINAIS}

Os estudos considerados no presente levantamento indicam que as diversas técnicas de massagem terapêutica infantil podem promover mudanças nos indicadores de estresse em bebês vivendo em ambientes de cuidados coletivos. Esses estudos foram distribuídos em três grupos de indicadores, a saber: escalas de dor, marcadores bioquímicos do estresse e sinais vitais. As massagens que mais se destacaram foram aquelas desenvolvidas por grupos de pesquisa e profissionais da área de saúde, observando-se a necessidade de novos estudos que abordem mais amiúde massagens infantis originadas dos conhecimentos tradicionais dos povos, tais como a Shantala indiana e a Yakson coreana. Uma vez que os estudos avaliados no presente 
levantamento foram realizados predominantemente em âmbito hospitalar possivelmente pelo apoio/aporte logístico que viabilizou/facilitou a mensuração dos indicadores de estresse ora eleitos, as lacunas observadas estimulam/incentivam futuros estudos da mesma natureza em ambientes de creche e de acolhimento institucional.

\section{AGRADECIMENTOS}

À Lilian de Jesus Fontel Cunha Donato, por ajudar na visualização de aspectos da planilha de dados que foram decisivos na segunda fase de seleção dos artigos utilizados no presente estudo.

À Angélica Homobono Nobre, pelas revisões deste texto e suas preciosas sugestões para o mesmo.

\section{REFERÊNCIAS}

1. ABDALLAH B, BADR LK, HAWWARI M. The efficacy of massage on short and long term outcomes in preterm infants. Infant Behavior and Development, 2013; 36(4), 662-669.

2. AGER A, BLAKE C, STARK L et al. Child protection assessment in humanitarian emergencies: Case studies from Georgia, Gaza, Haiti and Yemen. Child Abuse and Neglect, 2011; 35(12), 1045-1052.

3. BOWLBY J. Apego e Perda - 2. Separação, Angústia e Raiva. 4th ed. São Paulo: Martins Fontes, 2004; 536p.

4. CHIK YM, IP WY, CHOI KC. The Effect of Upper Limb Massage on Infants' Venipuncture Pain. Pain Management Nursing, 2017; 18(1), 50-57.

5. DIEGO MA, FIELD T, HERNANDEZ-REIF M. Vagal activity, gastric motility, and weight gain in massaged preterm neonates. Journal of Pediatrics, 2005; 147(1), 50-55.

6. DIEGO MA, FIELD T, HERNANDEZ-REIF M. Temperature increases in preterm infants during massage therapy. Infant Behavior and Development, 2008; 31(1), 149-152.

7. DIEGO MA, FIELD T, HERNANDEZ-REIF M. Procedural pain heart rate responses in massaged preterm infants. Infant Behavior and Development, 2009; 32(2), 226-229.

8. DIEGO MA, FIELD T, HERNANDEZ-REIF M. Preterm infant weight gain is increased by massage therapy and exercise via different underlying mechanisms. Early Human Development, 2014; 90(3), 137-140.

9. FERBER SG, FELDMAN R, KOHELET D et al. Massage therapy facilitates mother-infant interaction in premature infants. Infant Behavior and Development, 2005; 28(1), 74-81.

10. FIELD T, DIEGO M, HERNANDEZ-REIF M. Massage therapy research. Developmental Review, 2007; 27(1), 7589.

11. FIELD T, SCHANBERG SM, SCAFIDI F et al. Tactile Kinesthetic Stimulation Effects on Preterm Neonates. Pediatrics, 1986; 77(5), 654-658.

12. FOGAÇA MC, CARVALHO WB, PERES CA. Salivary cortisol as an indicator of adrenocortical function in healthy infants, using massage therapy. São Paulo Medical Journal = Revista Paulista de Medicina, 2005; 123(5), 215218.

13. FREITAS OM, LOPES EM, DO CÉU FIGUEIREDO M et al. Massagem no recém-nascido pré-termo: é um cuidado de enfermagem seguro? Revista Portuguesa de Saude Publica, 2010; 28(2), 187-198.

14. HATHAWAY EE, LUBERTO CM, BOGENSCHUTZ LH et al. Integrative care therapies and physiological and pain-related outcomes in hospitalized infants. Global Advances In Health and Medicine, 2015; 4(4), 32-37.

15. IM H, KIM E. Effect of Yakson and Gentle Human Touch versus usual care on urine stress hormones and behaviors in preterm infants: A quasi-experimental study. International Journal of Nursing Studies, 2009; 46(4), 450-458.

16. JAIN S, KUMAR P, McMILLAN DD. Prior leg massage decreases pain responses to heel stick in preterm babies. Journal of Paediatrics and Child Health, 2006; 42(9), 505-508.

17. LEBOYER F. SHANTALA - Uma arte tradicional - Massagem para bebês. 7th ed. São Paulo: Ground, 1995; $134 p$.

18. LIVINGSTON K, BEIDER S, KANT AJ et al. Touch and massage for medically fragile infants. Evidence-Based Complementary and Alternative Medicine : ECAM, 2009; 6(4), 473-482.

19. MORHENN V, BEAVIN LE, ZAK PJ. Massage increases oxytocin and reduces adrenocorticotropin hormone in humans. Alternative Therapies in Health and Medicine, 2012; 18(6), 11-18.

20. MOURA GG, AMORIM KS. A (in)visibilidade dos bebês na discussão sobre acolhimento institucional. Psicologia Em Estudo, 2013; 18(2), 235-245.

21. NEU M, PAN Z, WORKMAN R et al. Benefits of massage therapy for infants with symptoms of gastroesophageal 
reflux disease. Biological Research for Nursing, 2014; 16(4), 387-397.

22. OLIVEIRA BRG, VIEIRA CS, FURTADO MCC et al. Perfil de morbidade de crianças hospitalizadas em um hospital público: implicações para a Enfermagem. Revista Brasileira de Enfermagem, 2012; 65(4), 586-593.

23. OLIVEIRA BRG, VIERA CS, COLLET N et al. Causas de hospitalização no SUS de crianças de zero a quatro anos no Brasil. Revista Brasileira de Epidemiologia, 2010; 13(2), 268-277.

24. PICCININI CA, POLLI RG, BORTOLINI M et al. Razões maternas para colocar ou não o bebê na creche. Arquivos Brasileiros de Psicologia, 2016; 3(68), 59-74.

25. RAD Z, HAGHSHENAS M, JAVADIAN $Y$ et al. The effect of massage on weight gain in very low birth weight neonates. Journal of Clinical Neonatology, 2016; 5(2), 96.

26. RANGEY PS, SHETH M. Comparative Effect of Massage Therapy versus Kangaroo Mother Care on Body Weight and Length of Hospital Stay in Low Birth Weight Preterm Infants. International Journal of Pediatrics, 2014; 2014, $1-4$.

27. SILVA ERA. O direito à convivência familiar e comunitária - os abrigos para crianças e adolescentes no Brasil. Brasília: IPEA; 2004; 416p.

28. SMITH JR, MCGRATH J, BROTTO $M$ et al. A randomized-controlled trial pilot study examining the neurodevelopmental effects of a 5-week M Technique intervention on very preterm infants. Advances in Neonatal Care : Official Journal of the National Association of Neonatal Nurses, 2014; 14(3), 187-200.

29. SMITH SL, HALEY S, SLATER $\mathrm{H}$ et al. Heart rate variability during caregiving and sleep after massage therapy in preterm infants. Early Human Development, 2013; 89(8), 525-529.

30. SPITZ RA. O primeiro ano de vida. 3rd ed. São Paulo: Martins Fontes; 2004; 345p.

31. WHITE-TRAUT RC, SCHWERTZ D, MCFARLIN B et al. Salivary cortisol and behavioral state responses of healthy newborn infants to tactile-only and multisensory interventions. JOGNN - Journal of Obstetric, Gynecologic, and Neonatal Nursing, 2009; 38(1), 22-34.

32. YATES CC, MITCHELL AJ, BOOTH MY et al. The Effects of Massage Therapy to Induce Sleep in Infants Born Preterm. Pediatric Physical Therapy, 2014; 26, 405-410.

33. ZEANAH CH, NELSON CBA, FOX NA. Designing research to study the effects of institutionalization on brain and behavioral development: The Bucharest Early Intervention Project. Development \& Psychopathology, 2003; 15, 885-907. 